\title{
Assessment of intracranial vessels in association with carotid atherosclerosis and brain vascular lesions in rheumatoid arthritis
}

Csaba Oláh', Zsófia Kardos², Mariann Sepsi ${ }^{3}$, Attila Sas ${ }^{4}$, László Kostyál ${ }^{3}$, Harjit Pal Bhattoa ${ }^{5}$, Katalin Hodosi ${ }^{6}$, György Kerekes ${ }^{7}$, László Tamási², Attila Valikovics ${ }^{4}$, Dániel Bereczki ${ }^{8,9}$ and Zoltán Szekanecz ${ }^{6^{*}}$

\begin{abstract}
Background: Stroke has been associated with rheumatoid arthritis (RA). We assessed patients with RA and healthy control subjects by transcranial Doppler (TCD), carotid ultrasonography and brain magnetic resonance imaging (MRI).

Methods: Altogether, 41 female patients with RA undergoing methotrexate (MTX) or biologic treatment and 60 age-matched control subjects underwent TCD assessment of the middle cerebral artery (MCA) and basilar artery. Pulsatility index (PI), resistivity (resistance) index (RI) and circulatory reserve capacity (CRC) were determined at rest (r) and after apnoea (a) and hyperventilation (h). The presence of carotid plaques and carotid intima-media thickness (cIMT) were also determined. Intracerebral vascular lesions were investigated by brain MRI.

Results: MCA PI and RI values at rest and after apnoea were significantly increased in the total and MTX-treated RA populations vs control subjects. MCA CRC was also impaired, and basilar artery PI was higher in RA. More patients with RA had carotid plaques and increased cIMT. Linear regression analysis revealed that left PI(r) and RI(r) correlated with disease duration and that left $\mathrm{PI}(\mathrm{r}), \mathrm{RI}(\mathrm{r}), \mathrm{PI}(\mathrm{a}), \mathrm{PI}(\mathrm{h})$ and basilar PI correlated with disease activity. Right CRC inversely correlated with 28-joint Disease Activity Score. Disease activity was an independent determinant of left PI(a) and right CRC. Compared with long-term MTX treatment alone, the use of biologics in combination with MTX was associated with less impaired cerebral circulation. Impaired cerebral circulation was also associated with measures of carotid atherosclerosis.
\end{abstract}

Conclusions: To our knowledge, this is the first study to show increased distal MCA and basilar artery occlusion in RA as determined by TCD. Patients with RA also had CRC defects. We also confirmed increased carotid plaque formation and increased CIMT. Biologics may beneficially influence some parameters in the intracranial vessels.

Keywords: Rheumatoid arthritis, Cerebrovascular disease, Stroke, Transcranial Doppler, Carotid artery, Methotrexate, Biologic therapy

\footnotetext{
* Correspondence: szekanecz.zoltan@med.unideb.hu; http://www.rheumatology.hu

Csaba Oláh and Zsófia Kardos share first authorship as equal contributors.

Dániel Bereczki and Zoltán Szekanecz share last authorship as equal

contributors.

${ }^{6}$ Division of Rheumatology, Department of Medicine, Faculty of Medicine,

University of Debrecen, 98 Nagyerdei Street, H-4032 Debrecen, Hungary

Full list of author information is available at the end of the article
}

(c) The Author(s). 2017 Open Access This article is distributed under the terms of the Creative Commons Attribution 4.0 International License (http://creativecommons.org/licenses/by/4.0/), which permits unrestricted use, distribution, and reproduction in any medium, provided you give appropriate credit to the original author(s) and the source, provide a link to the Creative Commons license, and indicate if changes were made. The Creative Commons Public Domain Dedication waiver (http://creativecommons.org/publicdomain/zero/1.0/) applies to the data made available in this article, unless otherwise stated. 


\section{Background}

Rheumatoid arthritis (RA) is a chronic inflammatory rheumatic disease often involving the vascular and nervous systems [1]. Accelerated atherosclerosis and increased stroke morbidity and mortality have been associated with RA [2-5]. According to a recent metaanalysis, there is an excess risk of stroke (ORs ranging from 1.51 to 2.13) [5]. Yet, to date, no systematic evaluation of intracranial circulation has been performed.

Transcranial Doppler (TCD), first described in 1982, is a non-invasive ultrasound (US) technique used to measure cerebral blood flow velocity in the major intracranial arteries. It involves use of low-frequency $(<2 \mathrm{MHz}) \mathrm{US}$ waves to assess cerebral arteries through relatively thin bone windows. TCD allows dynamic monitoring of cerebral circulation with a high temporal resolution. It is relatively inexpensive, reproducible and portable [6, 7]. TCD may provide important information about the pathophysiology and prognosis of cerebrovascular ischemia [7]. When assessing the middle cerebral artery (MCA) by TCD, mean flow velocity (MFV), pulsatility index (PI) and the PI/MFV ratio are prognostic factors for recurrent vascular events [8]. Furthermore, asymptomatic MCA stenosis, which can be detected by TCD, may be an important risk for stroke [9].

One limitation of TCD is that approximately $8-20 \%$ of individuals have inadequate temporal acoustic windows (TAWs) [10]. There is an increased prevalence of TAW failure in females and in elderly people [10]. Very recently, we assessed TAW failure in patients with RA compared with control subjects and found that TAW failure was significantly more common among patients with RA (Z. Kardos, C. Oláh, M. Sepsi, A. Sas, L. Kostyál, T. Bóta, H. Bhattoa, K. Hodosi, G. Kerekes, L. Tamási, D. Bereczki, Z. Szekanecz, unpublished data, September 2017).

As described above, patients with RA are at very high risk for cardiovascular and cerebrovascular disease. $\mathrm{Nu}$ merous studies have been performed on cardiovascular disease and its surrogate markers. However, because stroke is the second most common vascular complication in RA, there is a great need to assess cerebrovascular risk as well. Because very little information is available with respect to intracranial vessels in RA, we sought to assess the following: (1) to determine circulation in the MCA, as well as in basilar arteries, by TCD to determine whether there are abnormalities in intracranial circulation in RA; (2) to assess the effects of antitumour necrosis factor $\alpha$ biologics vs methotrexate (MTX) treatment on TCD parameters; and (3) to correlate intracranial circulation with carotid atherosclerosis and brain vascular lesions. Therefore, we performed an extensive study using TCD in patients with RA undergoing MTX or biologic treatment and compared them with healthy control subjects. The functional TCD assessment included the determination of several functional parameters described later. We also assessed carotid atherosclerosis by US and performed brain magnetic resonance imaging (MRI) to visualise vascular lesions as well as signs of emollition and atrophy. To our knowledge, this is the first study to assess intracranial vessels in patients with RA by TCD in conjunction with carotid and brain examinations.

\section{Methods}

\section{Patients and control subjects}

Altogether, 41 patients with RA undergoing regular follow-up at the Semmelweis Teaching Hospital, Miskolc, Hungary, were recruited for the study. All these patients had accessible TAWs as determined before the TCD investigations. None of the patients with RA and control subjects had any previous vascular events. The major characteristics of these 41 patients are shown in Table 1. All patients were female, and their mean age was $58.2 \pm 9.7$ years. Their mean disease duration was $12.2 \pm$ 8.0 years. Altogether, $70 \%$ were immunoglobulin M (IgM) rheumatoid factor (RF)-positive, and 65\% were anti-cyclic citrullinated peptide 2 antibody (anti-CCP2)-positive. Among these patients, 12 were biologic-free. They had been receiving MTX for a mean of $6.2 \pm 5.8$ years in an average dose of $14.6 \pm 6.1 \mathrm{mg} /$ week. Altogether, 29 patients had been receiving biologics (15 infliximab [IFX] and 14 tocilizumab [TCZ]) as first-line biologic treatment, in combination with MTX, for a mean duration of $5.3 \pm 2.0$ years. The characteristics of the MTX- and biologic-treated RA subsets are shown in Table 1.

For this study, a cohort of 60 age-matched women without RA who were undergoing TCD investigations were chosen as the control group. These individuals were part of a TCD screening program conducted in the Miskolc region. The mean age of these 60 individuals was $58.4 \pm 4.4$ years. The age of control subjects was not significantly different from that of patients with RA.

Ethical approval (1046-63/2015) was obtained from the University of Miskolc Regional/Institutional Review Board. All patients signed informed consent forms. The study was performed according to the Declaration of Helsinki.

\section{Determination of temporal acoustic windows}

Before the TCD assessment was carried out by US, an ultrasonographer (MS) determined whether the right and left TAWs were detectable and available for TCD measurements. Only patients and control subjects with detectable TAWs could be further analysed by TCD.

\section{Transcranial Doppler assessment}

The Doppler effect means that if US waves strike a moving object, such as an erythrocyte, the reflected wave 
Table 1 General characteristics and laboratory markers of assessed patients with rheumatoid arthritis and control subjects

\begin{tabular}{lllll}
\hline & RA total & RA MTX & RA biologic & Control subjects \\
\hline No. of subjects & 41 & 12 & 29 & 60 \\
Age, years & $58.24 \pm 9.72$ & $58.67 \pm 9.88$ & $58.07 \pm 9.88$ & $58.42 \pm 4.41$ \\
Disease duration. years & $12.15 \pm 8.03$ & $11.00 \pm 8.63$ & $12.62 \pm 7.87$ & - \\
RF positivity, \% & $70 \%$ & $82 \%$ & $66 \%$ & - \\
Anti-CCP positivity, \% & $65 \%$ & $75 \%$ & $64 \%$ & - \\
MTX duration, years & $6.15 \pm 5.82$ & $5.83 \pm 4.57$ & $6.28 \pm 3.54$ & - \\
MTX dose, mg/week & $14.63 \pm 6.11$ & $14.17 \pm 5.15$ & $14.83 \pm 6.54$ & - \\
Biologic duration, years & $5.31 \pm 2.04$ & - & $5.31 \pm 2.04$ & - \\
DAS28 & $2.44 \pm 0.87$ & $2.88 \pm 0.75$ & $2.26 \pm 0.86$ & - \\
ESR, mm/h & $17.07 \pm 15.09$ & $17.00 \pm 9.16$ & $3.10 \pm 17.10$ & $5.30 \pm 4.70$ \\
hsCRP, mg/L & $3.93 \pm 4.53$ & $5.31 \pm 3.90$ & $28.51 \pm 5.02$ & $0.30 \pm 0.30$ \\
BMI, kg/m ${ }^{2}$ & $28.38 \pm 5.51$ & $28.08 \pm 6.77$ & $5.23 \pm 1.10$ & $30.20 \pm 8.70$ \\
TC, mmol/L & $5.34 \pm 1.08$ & $5.63 \pm 1.00$ & $1.49 \pm 0.40$ & $5.27 \pm 1.15$ \\
HDL-C, mmol/L & $1.47 \pm 0.43$ & $1.38 \pm 0.53$ & $3.73 \pm 1.20$ & $1.70 \pm 0.42$ \\
TC/HDL-C ratio & $3.94 \pm 1.36$ & $4.70 \pm 3.73$ & $3.12 \pm 0.81$ & $3.62 \pm 0.95$ \\
LDL-C, mmol/L & $3.17 \pm 0.82$ & $3.36 \pm 0.90$ & $1.43 \pm 0.66$ & $3.40 \pm 0.94$ \\
TG, mmol/L & $1.46 \pm 0.68$ & $1.53 \pm 0.76$ & $239.59 \pm 290.74$ & $1.83 \pm 0.53$ \\
Lp(a), ng/L & $260.14 \pm 317.20$ & $345.29 \pm 426.55$ & $1.71 \pm 0.49$ & - \\
ApoA/ApoB ratio & $1.81 \pm 0.51$ & & -
\end{tabular}

Abbreviations: Apo Apolipoprotein, BMI Body mass index, CCP Cyclic citrullinated peptide, CRC Cerebrovascular reserve capacity, DAS28 28-joint Disease Activity Score, ESR Erythrocyte sedimentation rate, HDL-C High-density lipoprotein cholesterol, $h s C R P$ High-sensitivity C-reactive protein, $L D L-C$ Low-density lipoprotein cholesterol, $L p($ a) Serum lipoprotein A, MTX Methotrexate, RA Rheumatoid arthritis, RF Rheumatoid factor, TC Total cholesterol, TG Triglyceride

undergoes a change in frequency (Doppler shift) which is directly proportional to the velocity of the reflector. A US frequency of $<2 \mathrm{MHz}$ is required to penetrate the skull and reach the intracranial vasculature. Acoustic windows are either foramina or thin bone that allow US waves to reach the cerebral circulation. The TAW located above the zygomatic ridge is most frequently used for detection of the anterior cerebral artery, MCA or posterior cerebral artery, as well as the terminal internal carotid artery (ICA) [6]. Unfortunately, as described above, approximately $8-20 \%$ of individuals [10] may have TAW failure.

In this study, MCAs were assessed by TCD using a Multi-Dop $\mathrm{T}$ Digital (DWL Compumedics $\mathrm{GmbH}$, Singen, Germany) device. Among TCD indices, MFV (cm/second), peak systolic flow velocity (PSFV) and end diastolic flow velocity (EDFV) are key parameters in TCD assessment. MFV is the mean velocity within a cardiac cycle. Increased MFV may indicate stenosis, vasospasm or hyperdynamic flow. A decreased value may indicate hypotension, decreased cerebral blood flow and intracranial pressure.

The PI, calculated as (PSFV - EDFV)/MFV, provides information on downstream cerebral vascular resistance (normal range 0.6-1.2). Proximal stenosis or occlusion may lower the PI below 0.5, whereas distal occlusion or constriction may increase the PI above 1.2. The resistivity (resistance) index (RI), calculated as (PSFV-EDFV)/ PSFV (normal range 0.4-0.7), indicates downstream resistance.

Cerebrovascular reserve capacity (CRC) is the physiological vasoconstrictive and vasodilatory function of precapillary (resistance) arteries that maintains optimal cerebral blood flow even if cerebral perfusion pressure changes. In the MCAs, CRC in percent is calculated as $(\mathrm{PSFV}-\mathrm{MFV}) / \mathrm{MFV} \times 100$. A normal response in $\mathrm{MFV}$ values after acetazolamide is a $29 \mathrm{~cm} /$ second increase in women and $21 \mathrm{~cm} /$ second increase in men [11], which corresponds to $49.3 \%$ and $38.9 \%$ CRC, respectively.

First, MFV, PI and RI were determined at rest (r) $[\operatorname{MFV}(r), \operatorname{PI}(r), \operatorname{RI}(\mathrm{r})]$. Then, provocation manoeuvres were performed, including hyperventilation (h) for 30 seconds [MFV(h), PI(h), RI(h)], followed by, after normal breathing, apnoea (a) for 30 seconds [MFV(a), $\mathrm{PI}(\mathrm{a}), \mathrm{RI}(\mathrm{a})$ ] [12]. After these manoeuvres, the flow velocity in MCA should increase by at least $15 \%$ under normal conditions; otherwise, severely impaired CRC is present [12].

The TCD procedure was performed by a single technician (MS) and validated by a neurosurgeon/ 
neuroradiologist $(\mathrm{CO})$. All the above-mentioned parameters were determined in the RA cohort. In control subjects, all parameters described above could also be assessed.

\section{Carotid artery ultrasound examination}

Carotid artery assessments were carried out in all patients with RA $(n=41)$ and control subjects $(n=60)$ using a duplex US system (Vivid e; GE Healthcare, Wauwatosa, WI, USA) using a 5-MHz linear array transducer (GE 8 L-RS probe; GE Healthcare) as described by us elsewhere [13]. Assessments were performed by a single observer (AS). In brief, five measurements were performed on the posterior wall of the distal part of common carotid artery (CCA), proximal to the carotid bulb. Longitudinal high-resolution B-mode US scans were employed over both the right and left CCAs and were R-synchronised and recorded. Carotid intimamedia thickness (cIMT) was defined as the distance between the first and second echogenic lines from the lumen, taking the average of five measurements on both sides. cIMT values were expressed in millimetres.

During US assessments, all visible plaques were detected and counted, and their characteristics (fibrous, calcified or soft, presence or absence of stenosis) were determined. Soft plaques representing unstable plaques are more dangerous than fibrous or calcified ones $[13,14]$. A 'plaque score' of $0-5$ was also assigned to each patient, with scores of $0-5$ representing no plaque, fibrous, non-stenotic calcified, stenotic calcified, soft and stenotic soft, respectively.

\section{Brain MRI investigations}

All 41 patients with RA also underwent brain MRI studies to be assessed for focal vascular lesions, emollition and atrophy. For this purpose, a MAGNETOM Verio 3 T MRI instrument (Siemens, Munich, Germany) was used. All MRI scans were obtained by a single radiologist (LK) and read by a neurosurgeon/neuroradiologist (CO). The presence or absence of vascular lesions, emollition and/or atrophy was noted.

\section{Laboratory assessments}

Serum IgM RF and high-sensitivity C-reactive protein (hsCRP) were assessed by quantitative nephelometry (COBAS MIRA Plus; Roche Diagnostics, Indianapolis, IN, USA), using RF and CRP reagents, respectively (both from DIALAB, Wiener Neudorf, Austria). RF levels $>50 \mathrm{IU} / \mathrm{ml}$ indicated seropositivity, and hsCRP levels $>5 \mathrm{mg} / \mathrm{L}$ were considered elevated. Anti-CCP autoantibodies were detected in serum samples using the second-generation Immunoscan-RA CCP2 enzyme-linked immunosorbent assay (Euro-Diagnostica, Arnhem, The Netherlands). The assay was performed according to the instructions of the manufacturer. A concentration $>25 \mathrm{IU} / \mathrm{ml}$ indicated seropositivity.

After overnight fasting, blood samples were taken from the patients and control subjects for total cholesterol (TC), low-density lipoprotein cholesterol (LDL-C), high-density lipoprotein cholesterol (HDL-C) and triglycerides. Lipids were determined using routine laboratory methods. Serum lipoprotein A [Lp(a)] was assessed by latex-sensitised immunoturbidimetry (Roche Diagnostics). Serum apolipoprotein A (ApoA) and ApoB levels were measured by immunoturbidimetry using Tina-quant Apoliporotein A and Apoliporotein B reagents (Roche Diagnostics) and a cobas Integra 700 analyser (Roche Diagnostics). ApoB/ApoA ratio, a good marker for atherosclerotic risk $[15,16]$, was calculated (Table 1).

\section{Statistical analysis}

The statistical analysis was performed with IBM SPSS Statistics version 22 software (IBM, Armonk, NY, USA). The data are expressed as mean $\pm \mathrm{SD}$ and as frequency and percentage. Continuous variables were evaluated by paired two-tailed $t$ tests and Wilcoxon's test. Nominal variables were compared between groups using the chisquare or Fisher's exact test, as appropriate. Simple correlations were determined by Spearman's correlation analysis. Multiple linear regression using the stepwise method was used to determine correlations and independent associations between parameters. TCD/carotid parameters were the dependent variables, and other clinical and laboratory parameters (e.g., age, disease duration, RF and anti-CCP positivity, MTX dose and duration, biologic therapy duration, 28-joint Disease Activity Score [DAS28], erythrocyte sedimentation rate, hsCRP, body mass index [BMI], lipids) were independent variables. The $\beta$ standardised linear coefficients showing linear correlations between two parameters were determined. The B regression coefficient (with 95\% CI) indicated an independent association between the dependent and independent variables during changes. $p$ values $<0.05$ were considered significant.

\section{Results}

\section{Comparative description of RA subsets}

With respect to clinical and laboratory characteristics (Table 1), the total RA cohort, MTX- and biologic-treated patients did not differ from each other in most respects. However, MTX-treated patients had significantly higher CRP $(5.3 \pm 3.9 \mathrm{mg} / \mathrm{L}$ vs $3.4 \pm 4.7 \mathrm{mg} / \mathrm{L} ; p<0.05)$ and mean DAS28 $(2.88 \pm 0.75$ vs $2.26 \pm 0.86 ; p=0.006)$ than any biologic-treated ones (Table 1). Numerous lipid parameters have been studied. Results showing no major differences between RA subsets are provided in Table 1 . 


\section{TCD assessments in patients with RA, RA subsets and control subjects}

Right and left MCA PI, RI and MFV values at rest (r) and after hyperventilation (h) and apnoea (a); right and left CRC values; and basilar artery $\mathrm{PI}(\mathrm{r})$ and $\operatorname{MFV}(\mathrm{r})$ values of patients with RA and control subjects have been evaluated and are presented. MCA PI and RI values detected at rest (r), after apnoea (a) and hyperventilation (h) are shown in Figs. 1 and 2. With respect to right and left MCA PI(r), all patients with RA (right $0.90 \pm 0.18$, $p<0.001$; left $0.89 \pm 0.19, p=0.003$ ) and MTX-treated patients (right $0.97 \pm 0.12, p<0.001$; left $1.00 \pm 0.19, p<0.001$ ) had significantly higher $\mathrm{PI}(\mathrm{r})$ values than control subjects (right $0.78 \pm 0.13$; left $0.77 \pm 0.44$ ) (Fig. 1a). Biologic-treated patients with RA exerted significantly higher left MCA $\mathrm{PI}(\mathrm{r})$ than control subjects $(0.83 \pm 0.17$ vs $0.77 \pm 0.44$, $p=0.021)$ but significantly lower left MCA PI(r) than MTX-treated patients $(0.83 \pm 0.17$ vs $1.00 \pm 0.19, p=0.021)$ (Fig. 1a).

With regard to right and left MCA PI(a), the total RA cohort (right $0.83 \pm 0.16, p<0.001$; left $0.85 \pm 0.03, p=0.002$ ) and MTX-treated patients (right $0.90 \pm 0.15, p<0.001$; left $0.90 \pm 0.15, p=0.009$ ) had significantly higher PI(a) values than control subjects (right $0.69 \pm 0.17$; left $0.68 \pm 0.25$ ) (Fig. 1b). On one hand, the total and MTX-treated RA population did not differ from control subjects in PI(h) values. On the other hand, biologic-treated patients with RA exerted significantly lower left MCA PI(h) than MTXtreated patients $(0.99 \pm 0.25$ vs $1.23 \pm 0.29, p=0.029)$ (Fig. 1c).
Right and left MCA RI(r) values were also significantly higher in the total (right $0.58 \pm 0.07, p<0.001$; left $0.58 \pm 0.08, p=0.004)$ and MTX-treated patients with RA (right $0.61 \pm 0.06, p<0.001$; left $0.62 \pm 0.07$, $p<0.001$ ) than control subjects (right $0.53 \pm 0.08$; left $0.53 \pm 0.07$ ) (Fig. 2a). Biologic-treated patients with RA had significantly higher left MCA RI(r) than control subjects $(0.55 \pm 0.07$ vs $0.53 \pm 0.07, p=0.018)$ but significantly lower left MCA RI(r) than MTX-treated patients $(0.55 \pm$ 0.07 vs $0.62 \pm 0.07, p=0.018$ ) (Fig. $2 \mathrm{a}$ ).

Right and left RI(a) was also significantly higher in all patients with RA (right $0.56 \pm 0.07, p<0.001$; left $0.56 \pm 0.02$, $p<0.001$ ) and in MTX-treated patients with RA (right $0.58 \pm 0.06, p<0.001$; left $0.59 \pm 0.07, p<0.001)$ than in control subjects (right $0.49 \pm 0.08$; left $0.49 \pm 0.07$ ) (Fig. 2b). Finally, patients with RA did not differ from control subjects in right MCA RI(h) values. However, MTX-treated patients with RA exerted significantly higher left MCA $\mathrm{RI}(\mathrm{h})$ than control subjects $(0.69 \pm 0.07$ vs $0.62 \pm 0.29$, $p=0.09$ ) (Fig. 2c). Basilar artery $\mathrm{PI}(\mathrm{r})$ was also increased in the total RA cohort $(0.98 \pm 0.26, p=0.005)$ and in MTX-treated patients $(1.05 \pm 0.38, p=0.001)$ compared with control subjects $(0.85 \pm 0.15)$ (Fig. 3$)$.

The right and left CRC was significantly impaired in all patients with RA (right $27.5 \pm 12.5 \%, p<0.001$; left $27.3 \pm 13.5, p<0.001)$, MTX-treated patients with RA (right 24.5 $\pm 9.8, p=0.005$; left 25.6 $\pm 7.6, p=0.002$ ) and biologic-treated patients with RA (right $28.8 \pm 13.5$, $p<0.001$; left $28.2 \pm 15.9, p<0.001$ ) compared with control subjects (right 50.2 \pm 30.6 ; left $44.0 \pm 17.6$ ) (Fig. 4).

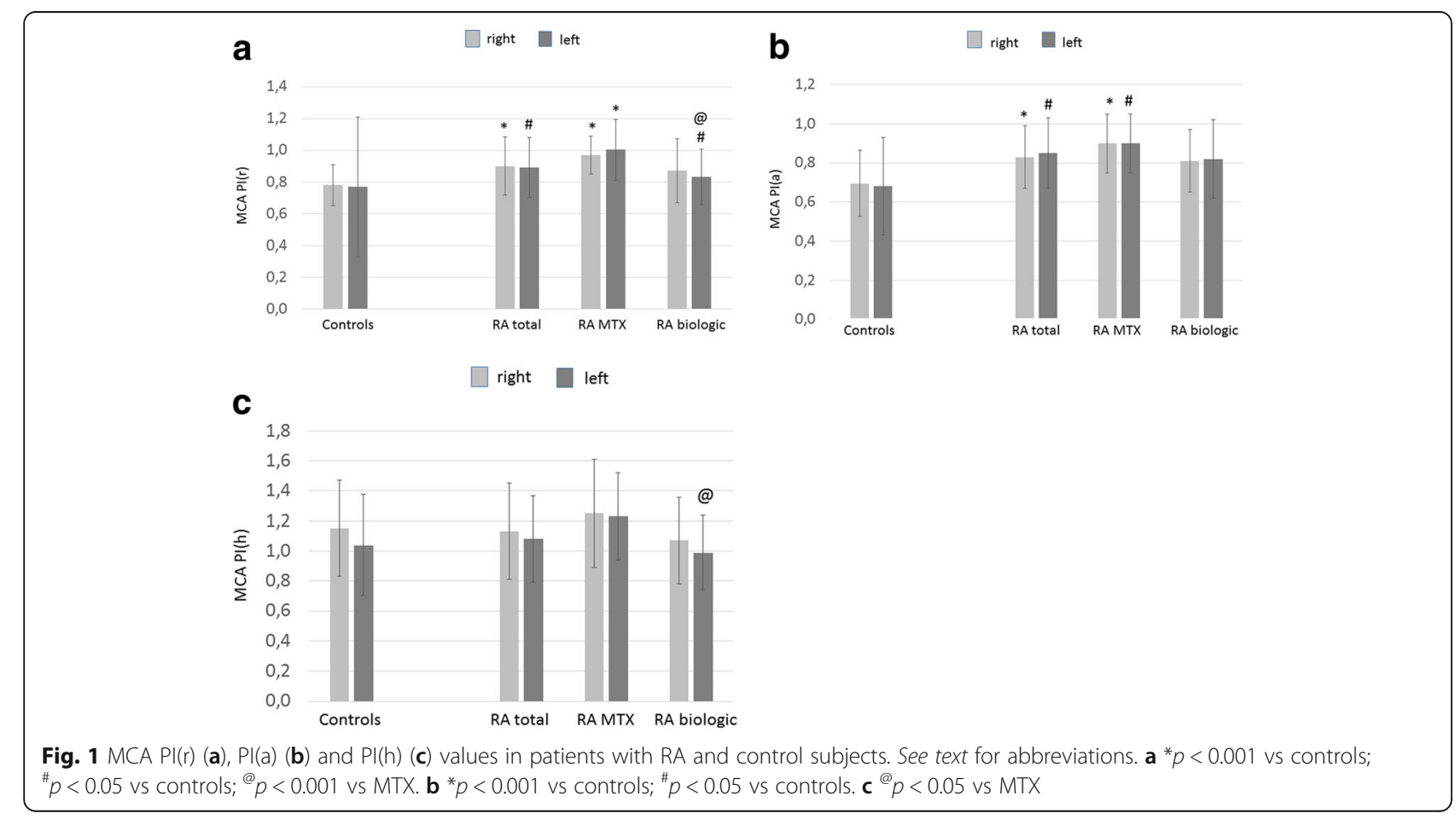



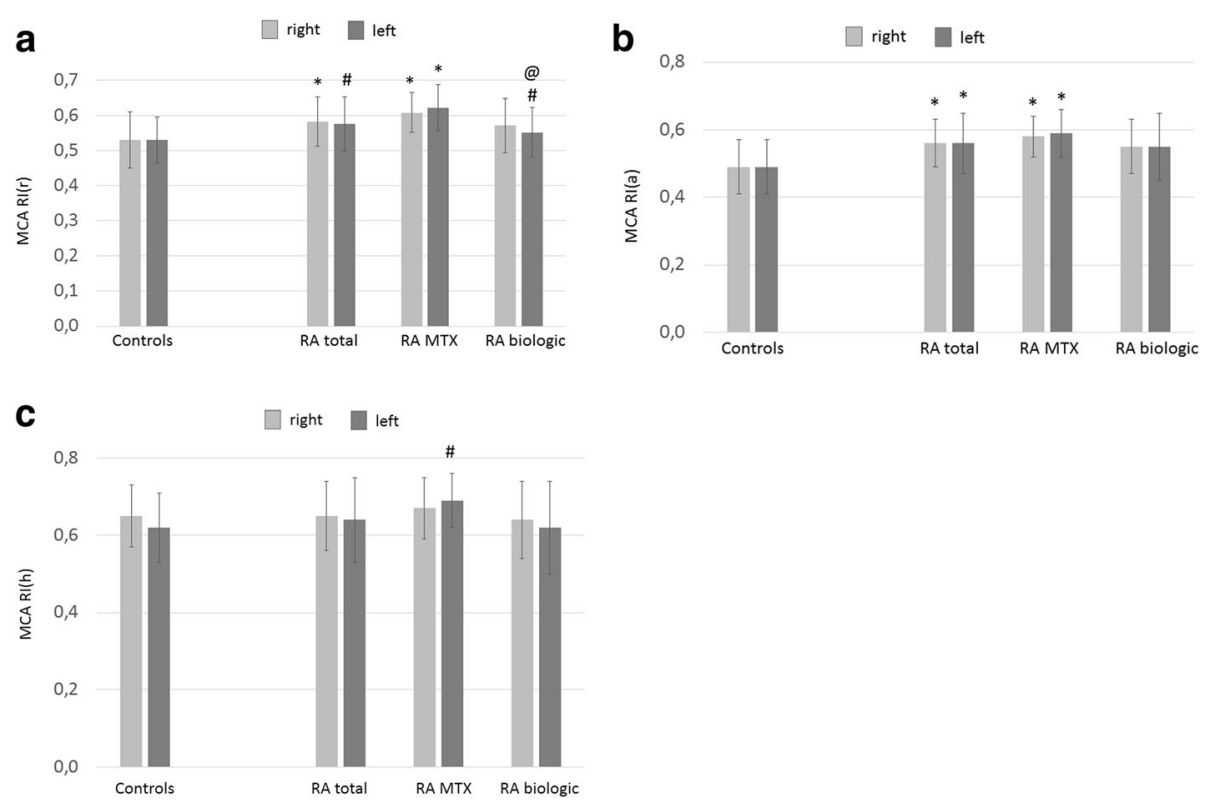

Fig. 2 MCA RI(r) (a), RI(a) (b) and RI(h) (c) values in patients with RA and control subjects. See text for abbreviations. a * $p<0.001$ vs controls; ${ }^{\#} p<0.05$ vs controls; ${ }^{\circledR} p<0.05$ vs MTX $\mathbf{b}{ }^{*} p<0.001$ vs controls $\mathbf{c}{ }^{*} p<0.05$ vs controls

Finally, in addition to the significant differences described above, right MCA PI(r), PI(a), PI(h), RI(r), RI(a), RI(h), left MCA PI(a), RI(a), RI(h), basilar artery $\mathrm{PI}(\mathrm{r})$ values exerted a tendency of being increased in MTX-treated vs biologic-treated patients (Figs. 1, 2 and 3). Also, right and left MCA CRC was nonsignificantly lower in MTX-treated than in biologic-treated patients (Fig. 4).

\section{Carotid artery assessments}

The right and left carotid arteries could be assessed in all patients with RA $(n=41)$ and control subjects $(n=60)$. With respect to the right carotid, the percentage of patients with at least one plaque was significantly higher in all patients with RA $(51 \% ; p=0.021)$ and MTX-treated subjects $(67 \% ; p=0.016)$ than in control subjects $(27 \%)$.

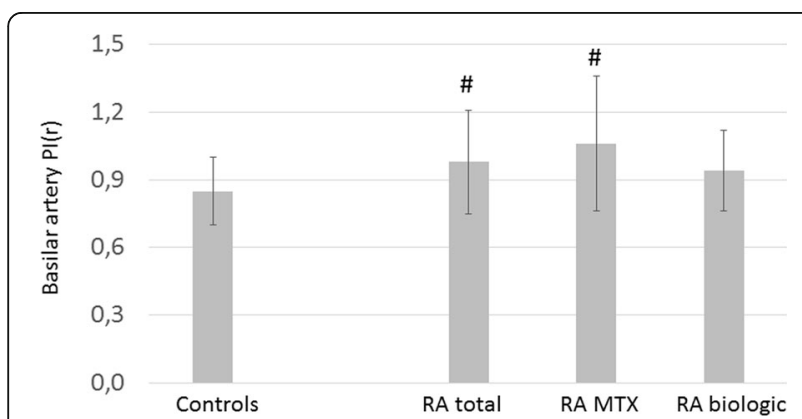

Fig. 3 Basilar artery PI(r) values in patients with RA and control subjects. See text for abbreviations. ${ }^{*} p<0.05$ vs controls
There was no difference between the subsets with regard to left carotid plaque percentages (Table 2). Likewise, the right but not the left carotid plaque score as defined above was also significantly higher in the total $(1.22 \pm 1.35$; $p=0.016)$ and MTX-treated RA subset $(1.58 \pm 1.31$; $p=0.08$ ) (Table 2).

The cIMT of the right carotid artery was $0.72 \pm 0.24 \mathrm{~mm}$ in the total RA cohort and $0.40 \pm 0.28 \mathrm{~mm}$ in control subjects $(p<0.001)$. Similarly, the left carotid cIMT was greater in RA $(0.73 \pm 0.23 \mathrm{~mm})$ than in control subjects $(0.40 \pm 0.28 \mathrm{~mm})(p<0.001)$. Right and left cIMT were also significantly higher in MTX-treated (right $0.72 \pm 0.25 \mathrm{~mm}$, $p<0.001$; left $0.69 \pm 0.22 \mathrm{~mm}, p<0.001)$ and biologictreated patients (right $0.72 \pm 0.23, p<0.001$; left $0.75 \pm$ $0.24 \mathrm{~mm} 0, p<0.001$ ) than in control subjects (right $0.40 \pm$ $0.28 \mathrm{~mm}$; left $0.40 \pm 0.28 \mathrm{~mm}$ ) (Table 2).

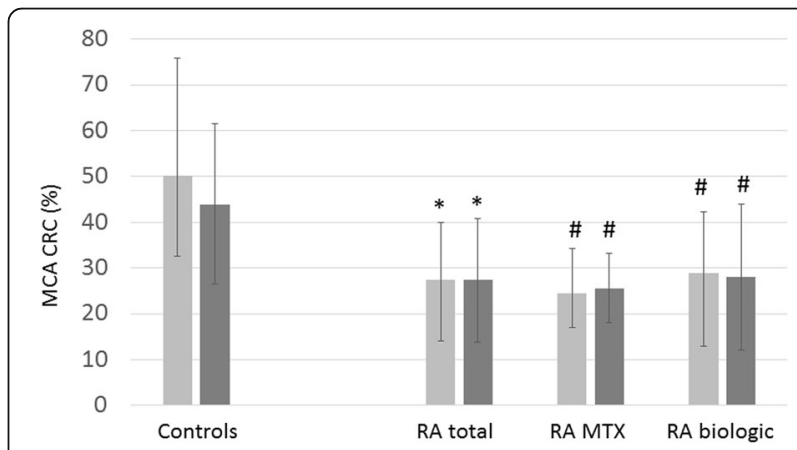

Fig. 4 MCA CRC values in patients with RA and control subjects. See text for abbreviations. ${ }^{*} p<0.001$ vs controls; ${ }^{*} p<0.05$ vs controls 
Table 2 Carotid intima-media thickness and brain magnetic resonance imaging assessments

\begin{tabular}{|c|c|c|c|c|c|c|c|c|}
\hline & \multirow{2}{*}{$\begin{array}{l}\text { RA total } \\
(n=41)\end{array}$} & \multirow{2}{*}{$\begin{array}{l}\text { RA MTX } \\
(n=12)\end{array}$} & \multirow{2}{*}{$\begin{array}{l}\text { RA biologic } \\
(n=29)\end{array}$} & \multirow{2}{*}{$\begin{array}{l}\text { Control subjects } \\
(n=60)\end{array}$} & \multicolumn{4}{|l|}{$p$ Value } \\
\hline & & & & & $\begin{array}{l}\text { RA total vs } \\
\text { control subjects }\end{array}$ & $\begin{array}{l}\text { RA MTX vs } \\
\text { control subjects }\end{array}$ & $\begin{array}{l}\text { RA biologic vs } \\
\text { control subjects }\end{array}$ & $\begin{array}{l}\text { RA MTX vs } \\
\text { RA biologic }\end{array}$ \\
\hline \multicolumn{9}{|l|}{ Carotid ultrasound studies } \\
\hline Right cIMT (mm) & $0.72 \pm 0.24$ & $0.72 \pm 0.25$ & $0.72 \pm 0.23$ & $0.40 \pm 0.28$ & $<0.001$ & $<0.001$ & $<0.001$ & \\
\hline Left cIMT (mm) & $0.73 \pm 0.23$ & $0.69 \pm 0.22$ & $0.75 \pm 0.24$ & $0.40 \pm 0.28$ & $<0.001$ & $<0.001$ & $<0.001$ & \\
\hline Right carotid plaques (\%) & $21(51 \%)$ & $8(67 \%)$ & $13(45 \%)$ & $16(27 \%)$ & 0.021 & 0.016 & & \\
\hline Left carotid plaques (\%) & $18(44 \%)$ & $6(50 \%)$ & $12(41 \%)$ & $21(35 \%)$ & & & & \\
\hline $\begin{array}{l}\text { Right carotid plaque } \\
\text { score }\end{array}$ & $1.22 \pm 1.35$ & $1.58 \pm 1.31$ & $1.07 \pm 1.36$ & $0.60 \pm 0.99$ & 0.016 & 0.008 & & \\
\hline $\begin{array}{l}\text { Left carotid plaque } \\
\text { score }\end{array}$ & $1.00 \pm 1.16$ & $1.25 \pm 1.55$ & $0.90 \pm 0.98$ & $0.78 \pm 1.12$ & & & & \\
\hline \multicolumn{9}{|l|}{ Brain MRI studies } \\
\hline Right vascular lesions (\%) & $16(39 \%)$ & $5(42 \%)$ & $11(38 \%)$ & & & & & \\
\hline Left vascular lesions (\%) & $16(39 \%)$ & $5(42 \%)$ & $11(38 \%)$ & & & & & \\
\hline Emollition & $2(5 \%)$ & 0 & $2(7 \%)$ & & & & & \\
\hline Atrophy & $8(20 \%)$ & $1(8 \%)$ & $7(24 \%)$ & & & & & \\
\hline
\end{tabular}

Abbreviations: CIMT Carotid intima-media thickness, MRI Magnetic resonance imaging, MTX Methotrexate, RA Rheumatoid arthritis

\section{Brain MRI investigations}

As described above, 3-T brain MRI investigations were carried out in the 41 patients with RA. Altogether, 39\% of all patients with RA, $42 \%$ of MTX- and 38\% of biologic-treated patients with RA had at least one vascular lesion in the right and left cerebral hemispheres. Only two patients had signs of emollition, whereas eight patients had cerebral atrophy. There were no statistical differences between MTX- and biologic-treated patients (Table 2).

\section{Associations of TCD, carotid features and other parameters in patients with RA}

As described above, multiple linear regression analysis was performed to determine associations between TCD/ carotid parameters as dependent variables and other independent clinical and laboratory variables. As shown in Table 3, numerous TCD variables correlated with age. When assessing disease-related factors, left MCA PI(r) and $\mathrm{PI}(\mathrm{h})$ correlated positively, whereas right MCA CRC inversely correlated, with disease activity. With respect to metabolic factors, right $\mathrm{PI}(\mathrm{h})$ and right cIMT correlated with BMI. Left RI(h) inversely correlated with HDL-C.

We also correlated TCD variables with carotid US and brain MRI results. When comparing patients with RA with $(n=18)$ and without $(n=23)$ left carotid plaques, we found that right MCA PI(a), RI(r) and RI(a), as well as left MCA PI(a), PI(h) and RI(a), values were significantly higher in patients with carotid plaques (Table 4). In addition, right $\mathrm{MCA} \mathrm{PI}(\mathrm{h})$ significantly correlated with right $\operatorname{cIMT}(R=0.377, p=0.018)$ (data not shown).
TCD and carotid parameters did not show significant associations with any brain MRI findings (data not shown).

\section{Discussion}

RA has been associated with accelerated atherosclerosis leading to increased cardiovascular and cerebrovascular morbidity and mortality $[2-5,17,18]$. Cerebrovascular disease and stroke may occur with uncontrolled, more severe disease $[5,17-19]$. It is very important to assess

Table 3 Associations between transcranial Doppler/carotid parameters and other variables ${ }^{a}$

\begin{tabular}{|c|c|c|c|c|}
\hline Parameter 1 & Parameter 2 & B $(95 \%$ Cl) & $\beta$ & $p$ Value \\
\hline Right $\mathrm{PI}(\mathrm{r})$ & Age & $0.007(0.002-0.013)$ & 0.384 & 0.011 \\
\hline Right $R I(r)$ & Age & $0.004(0.002-0.006)$ & 0.508 & 0.001 \\
\hline Right PI(h) & $\mathrm{BMI}$ & $0.024(0.006-0.042)$ & 0.410 & 0.011 \\
\hline Right RI(h) & Age & $0.004(0.002-0.007)$ & 0.456 & 0.002 \\
\hline Left PI(r) & Age & $0.009(0.002-0.017)$ & 0.447 & 0.012 \\
\hline Left RI(r) & Age & $0.004(0.001-0.007)$ & 0.459 & 0.011 \\
\hline Left PI(a) & DAS28 & $0.086(0.013-0.159)$ & 0.368 & 0.023 \\
\hline \multirow[t]{2}{*}{ Left PI(h) } & CRP & $0.028(0.005-0.050)$ & 0.442 & 0.016 \\
\hline & DAS28 & $0.087(0.008-0.166)$ & 0.372 & 0.032 \\
\hline Left RI(h) & $\mathrm{HDL}-\mathrm{C}$ & $-0.104(-0.203-0.006)$ & -0.415 & 0.039 \\
\hline Basilar PI & Age & $0.008(0.002-0.014)$ & 0.325 & 0.010 \\
\hline Right CRC & DAS28 & $-4.633(-9.047-0.219)$ & -0.326 & 0.040 \\
\hline Right cIMT & $\mathrm{BMI}$ & $0.002(0-00.3)$ & 0.306 & 0.015 \\
\hline
\end{tabular}

Abbreviations: $\beta$ Standardised linear coefficient, $B(+95 \%$ Cl) Regression coefficient, $B M I$ Body mass index, $C R P C$-reactive protein, $C R C$ Cerebrovascular reserve capacity, DAS28 28-joint Disease Activity Score, HDL-C High-density lipoprotein cholesterol, $P /$ Pulsatility index, $R I$ Resistivity (resistance) index aMultiple linear regression 
Table 4 Associations between transcranial Doppler and left carotid artery plaques

\begin{tabular}{lccc}
\hline TCD variable & $\begin{array}{l}\text { Presence of } \\
\text { plaque }(n=18)\end{array}$ & $\begin{array}{l}\text { Absence of } \\
\text { plaque }(n=23)\end{array}$ & $p$ Value \\
\hline Right PI(a) & $0.92 \pm 0.17$ & $0.77 \pm 0.12$ & 0.002 \\
Right RI(r) & $0.61 \pm 0.07$ & $0.56 \pm 0.07$ & 0.021 \\
Right RI(a) & $0.59 \pm 0.09$ & $0.53 \pm 0.05$ & 0.007 \\
Left PI(a) & $0.94 \pm 0.18$ & $0.79 \pm 0.17$ & 0.032 \\
Left PI(h) & $1.23 \pm 0.29$ & $0.94 \pm 0.25$ & 0.021 \\
Left RI(a) & $0.61 \pm 0.10$ & $0.53 \pm 0.07$ & 0.022 \\
\hline
\end{tabular}

Abbreviations: PI Pulsatility index, RI Resistivity (resistance) index, TCD Transcranial Doppler

the intracranial vessels as well as the carotid arteries in order to determine cerebrovascular risk and the preclinical status of patients $[14,19,20]$. To our knowledge, to date, no systematic evaluation of intracranial circulation using functional TCD has been performed. In addition, we believe this is the first study including use of TCD, assessment of carotid arteries and brain MRI in the very same RA cohort. Because of the lack of literature data, we could not compare our findings with data reported by others.

TCD allows dynamic monitoring of cerebral circulation [6]. TCD is a very convenient, cheap and portable technique that can be used at the bedside. TCD may be able to assess the pathophysiology and outcome of cerebral ischemia [7]. Moreover, MCA may be the most relevant vessel to study by TCD. MCA stenosis, as well as MCA PI, MFV and PI/MFV ratio, has been associated with stroke, and all are important predictors of future cerebrovascular events $[8,9]$.

TAW failure may limit the performance of TCD. Approximately $8-20 \%$ of people in the general population have undetectable TAW [10]. There has been no report on TAW failure in patients with RA. Very recently, we have found that TAW failure was significantly more common in RA than among the general population (Kardos et al., submitted). Therefore, before performing TCD measurements, we looked for right and left TAWs. Eventually, 41 consecutive patients were suitable for TCD, carotid artery and brain MRI studies.

We performed a detailed TCD assessment in a homogeneous RA population and with age-matched, healthy control subjects. We also compared patients treated with MTX and biologics including IFX and TCZ. The total, MTX-treated and biologic-treated patients did not differ from each other in most respects. MTX-treated patients had higher disease activity than biologic-treated patients.

With respect to MCA, PI and RI at rest and after apnoea measured on both sides were significantly higher in RA, indicating increased distal occlusion and resistance, respectively. Patients with $\mathrm{RA}$ also had an impaired
CRC, as indicated by lower CRC. Significantly higher PI was detected in the basilar artery as well. With regard to treatment, MTX-treated patients had higher PI and RI values than biologic-treated patients. For example, left MCA PI(r), PI(h) and RI(r) were significantly lower in biologic-treated patients than in MTX-treated patients. Furthermore, right $\mathrm{MCA} \mathrm{PI}(\mathrm{r})$ and $\mathrm{RI}(\mathrm{r})$, as well as right and left MCA PI(a) and RI(a), were still increased in MTX-treated patients compared with control subjects, whereas these parameters were similar to those of biologic-treated patients. These data suggest that patients with RA have impaired circulation in the MCA and basilar arteries compared with control subjects. Furthermore, biologics may improve numerous MCA parameters to a greater extent than MTX.

The carotid arteries were also assessed for signs of atherosclerosis. More patients with RA than control subjects had at least one plaque in the right carotid artery, and right carotid plaque score was also higher in patients with RA. There were no differences among RA subsets regarding the presence of plaques or plaque scores. The cIMT of the right and left carotid arteries was also much higher in the total, MTX-treated and biologic-treated patients with RA than in control subjects. We and others have previously reported increased cIMT and plaques in RA [13, 20-25]. In this cohort, the differences in cIMT between patients with RA and control subjects are really large, probably due to the fact that mostly patients with severe RA who needed biologics were selected. Carotid arteries have not yet been studied in conjunction with TCD in patients with RA.

Brain MRI investigations were carried out to determine cerebral vascular lesions. About half of patients with RA developed vascular lesions in the right or left hemisphere. Somewhat greater proportions of MTXtreated than biologic-treated patients who developed vascular lesions. We also detected signs of cerebral emollition and atrophy in about 5\% and 24\%, respectively, of patients with RA. We have not found any reports with respect to cerebral emollition in RA. Wartolowska et al. [26] also detected some atrophy in patients with RA. Bekkelund et al. [27] performed a quantitative cerebral MRI study in 1995. They did not find any differences in the prevalence of atrophy in patients with RA and control subjects [27].

Multiple linear regression analysis suggested the associations of multiple TCD parameters with age. Some parameters also correlated with RA disease activity. When TCD and carotid assessment results were correlated, numerous TCD variables were increased in patients with left carotid plaques. Right PI(h) also correlated with right cIMT. These correlations should be confirmed in larger future studies. However, comparative TCD, carotid US and brain MRI studies have not yet been performed in patients with RA. 


\section{Conclusions}

To our knowledge, this is the first detailed study using TCD to assess intracranial arteries in RA compared with control subjects. We also included carotid assessment and brain MRI in the same patients. We found more pronounced distal occlusion and resistance of the MCA along with impaired CRC in patients with RA compared with control subjects. We confirmed that carotid plaques and increased cIMT occur in patients with RA, and we described cerebral vascular lesions, emollition and atrophy in a group of patients with RA. With respect to therapy, biologic treatment, in comparison with MTX, may have more pronounced effects on some TCD PI and RI indices. Preclinical cerebrovascular screening may be important in determining stroke risk in individuals with RA.

\section{Key messages}

- Increased distal MCA and basilar artery occlusion was detected in patients with RA, as determined by TCD.

- Patients with RA also have CRC defects.

- Biologics may beneficially influence some parameters in the intracranial vessels.

\begin{abstract}
Abbreviations
Apo: Apolipoprotein; BMI: Body mass index; CCA: Common carotid artery; CCP: Cyclic citrullinated peptide; CIMT: Carotid intima-media thickness; CRP: Creactive protein; CRC: Cerebrovascular reserve capacity; DAS28: 28-joint Disease Activity Score; EDFV: End diastolic flow velocity; ESR: Erythrocyte sedimentation rate; HDL-C: High-density lipoprotein cholesterol; hsCRP: High-sensitivity C-reactive protein; IFX: Infliximab; IgM: Immunoglobulin M; LDL-C: Low-density lipoprotein cholesterol; Lp(a): Serum lipoprotein A; MCA: Middle cerebral artery; MFV: Mean flow velocity; MRI: Magnetic resonance imaging; MTX: Methotrexate; PI: Pulsatility index; PSFV: Peak systolic flow velocity; RA: Rheumatoid arthritis; RF: Rheumatoid factor; RI: Resistivity (resistance) index; TAW: Temporal acoustic window; TC: Total cholesterol; TCD: Transcranial Doppler; TCZ: Tocilizumab; TG: Triglyceride; US: Ultrasound
\end{abstract}

\section{Acknowledgements}

This work was supported by a K10073 grant (HPB and ZS) donated by the Hungarian Scientific Research Fund (OTKA), by European Union and the State of Hungary co-financing via the European Social Fund in the framework of TAMOP-4.2.4.A/2-11/1-2012-0001 'National Excellence Program' (ZS), and by European Union grants GINOP-2.3.2-15-2016-00015 and GINOP2.3.2-15-2016-00050 (ZS).

\section{Funding}

This work was supported by a K10073 grant (HPB and ZS) donated by the Hungarian Scientific Research Fund (OTKA), by European Union and the State of Hungary co-financing via the European Social Fund in the framework of TAMOP-4.2.4.A/2-11/1-2012-0001 'National Excellence Program' (ZS), and by European Union grants GINOP-2.3.2-15-2016-00015 and GINOP-2.3.2-15-2016-00050 (ZS).

Availability of data and materials Not applicable.

\section{Authors' contributions}

CO was responsible for study design, patient examination and manuscript drafting. ZK was responsible for patient enrolment, patient examination, data analysis and manuscript corrections. MS was responsible for TCD assessments and data analysis. AS and LK were responsible for TCD and CIMT assessments as well as data analysis. HPB was responsible for bone marker assays and bone data analysis. KH was responsible for data analysis, statistical expertise and manuscript drafting. GK was responsible for patient examination, CIMT assessments and data analysis. LT was responsible for patient enrolment and patient examination. AV was responsible for patient examination and manuscript drafting. DB was responsible for expert advice and manuscript drafting. ZS was responsible for expert advice, study design and manuscript drafting. All authors read and approved the final manuscript.

\section{Ethics approval and consent to participate}

Ethical approval (1046-63/2015) was obtained from the Regional/Institutional Review Board of Miskolc University. The study was performed according to the principles of the Declaration of Helsinki.

\section{Consent for publication}

No data that could identify individual patients are presented; therefore, consent was not needed.

\section{Competing interests}

The authors declare that they have no competing interests.

\section{Publisher's Note}

Springer Nature remains neutral with regard to jurisdictional claims in published maps and institutional affiliations.

\section{Author details}

'Department of Neurosurgery, Borsod County Teaching Hospital, Miskolc, Hungary. ${ }^{2}$ Department of Rheumatology, Borsod County Teaching Hospital, Miskolc, Hungary. ${ }^{3}$ Department of Radiology, Borsod County Teaching Hospital, Miskolc, Hungary. ${ }^{4}$ Department of Neurology, Borsod County Teaching Hospital, Miskolc, Hungary. ${ }^{5}$ Department of Laboratory Medicine, Faculty of Medicine, University of Debrecen, Debrecen, Hungary. ${ }^{6}$ Division of Rheumatology, Department of Medicine, Faculty of Medicine, University of Debrecen, 98 Nagyerdei Street, H-4032 Debrecen, Hungary. ${ }^{7}$ Department of Angiology, University of Debrecen Faculty of Medicine, Debrecen, Hungary. ${ }^{8}$ Department of Neurology, University of Debrecen Faculty of Medicine, Debrecen, Hungary. 'Department of Neurology, Semmelweis University, Budapest, Hungary.

Received: 6 July 2017 Accepted: 5 September 2017

Published online: 26 September 2017

\section{References}

1. Symmons DP. Epidemiology of rheumatoid arthritis: determinants of onset, persistence and outcome. Best Pract Res Clin Rheumatol. 2002;16(5):707-22.

2. Sodergren A, Stegmayr B, Ohman ML, Wallberg-Jonsson S. Increased incidence of stroke and impaired prognosis after stroke among patients with seropositive rheumatoid arthritis. Clin Exp Rheumatol. 2009;27(4):641-4.

3. Shoenfeld Y, Gerli R, Doria A, Matsuura E, Cerinic MM, Ronda N, Jara LJ, Abu-Shakra M, Meroni PL, Sherer Y. Accelerated atherosclerosis in autoimmune rheumatic diseases. Circulation. 2005;112(21):3337-47.

4. Szekanecz Z, Kerekes G, Végh E, Kardos Z, Baráth Z, Tamási L, Shoenfeld Y. Autoimmune atherosclerosis in 3D: how it develops, how to diagnose and what to do. Autoimmun Rev. 2016;15(7):756-69.

5. Wiseman SJ, Ralston SH, Wardlaw JM. Cerebrovascular disease in rheumatic diseases: a systematic review and meta-analysis. Stroke. 2016:47(4):943-50.

6. Guan J, Zhang S, Zhou Q, Li C, Lu Z. Usefulness of transcranial Doppler ultrasound in evaluating cervical-cranial collateral circulations. Interv Neuroradiol. 2013;2(1):8-18.

7. Sharma VK, Paliwal PR, Teoh HL, Venketasubramanian N, Chan BP. Role of diagnostic ultrasound in patient selection for stroke intervention. Recent Pat CNS Drug Discov. 2011;6(3):181-95.

8. Wijnhoud AD, Koudstaal PJ, Dippel DW. The prognostic value of pulsatility index, flow velocity, and their ratio, measured with TCD ultrasound, in patients with a recent TIA or ischemic stroke. Acta Neurol Scand. 2011;124(4):238-44.

9. Ni J, Yao M, Gao S, Cui LY. Stroke risk and prognostic factors of asymptomatic middle cerebral artery atherosclerotic stenosis. J Neurol Sci. 2011;301(1-2):63-5.

10. Marinoni M, Ginanneschi A, Forleo P, Amaducci L. Technical limits in transcranial Doppler recording: inadequate acoustic windows. Ultrasound Med Biol. 1997;23(8):1275-7.

11. Pindzola RR, Balzer JR, Nemoto EM, Goldstein S, Yonas H. Cerebrovascular reserve in patients with carotid occlusive disease assessed by stable 
xenon-enhanced $C T$ cerebral blood flow and transcranial Doppler. Stroke. 2001;32(8):1811-7.

12. Malatino LS, Bellofiore S, Costa MP, Lo Manto G, Finocchiaro F, Di Maria GU. Cerebral blood flow velocity after hyperventilation-induced vasoconstriction in hypertensive patients. Stroke. 1992;23(12):1728-32.

13. Kerekes G, Szekanecz Z, Der H, Sandor Z, Lakos G, Muszbek L, Csipo I, Sipka S, Seres I, Paragh G, et al. Endothelial dysfunction and atherosclerosis in rheumatoid arthritis: a multiparametric analysis using imaging techniques and laboratory markers of inflammation and autoimmunity. J Rheumatol. 2008;35(3):398-406.

14. Kerekes G, Soltesz P, Nurmohamed MT, Gonzalez-Gay MA, Turiel M, Vegh E, Shoenfeld Y, McInnes I, Szekanecz Z. Validated methods for assessment of subclinical atherosclerosis in rheumatology. Nat Rev Rheumatol. 2012;8(4):224-34

15. Garcia-Gomez C, Nolla JM, Valverde J, Gomez-Gerique JA, Castro MJ, Pinto X. Conventional lipid profile and lipoprotein(a) concentrations in treated patients with rheumatoid arthritis. J Rheumatol. 2009:36(7):1365-70.

16. Park YB, Lee SK, Lee WK, Suh CH, Lee CW, Lee CH, Song CH, Lee J. Lipid profiles in untreated patients with rheumatoid arthritis. J Rheumatol. 1999;26(8):1701-4.

17. Wolfe F, Freundlich B, Straus WL. Increase in cardiovascular and cerebrovascular disease prevalence in rheumatoid arthritis. J Rheumatol. 2003;30(1):36-40

18. Levy L, Fautrel B, Barnetche T, Schaeverbeke T. Incidence and risk of fatal myocardial infarction and stroke events in rheumatoid arthritis patients: a systematic review of the literature. Clin Exp Rheumatol. 2008;26(4):673-9.

19. Zha AM, Di Napoli M, Behrouz R. Prevention of stroke in rheumatoid arthritis. Curr Neurol Neurosci Rep. 2015;15(12):77.

20. Peters MJ, Symmons DP, McCarey D, Dijkmans BA, Nicola P, Kvien TK, McInnes IB, Haentzschel H, Gonzalez-Gay MA, Provan S, et al. EULAR evidence-based recommendations for cardiovascular risk management in patients with rheumatoid arthritis and other forms of inflammatory arthritis. Ann Rheum Dis. 2010:69(2):325-31.

21. Gonzalez-Juanatey C, Llorca J, Martin J, Gonzalez-Gay MA. Carotid intima-media thickness predicts the development of cardiovascular events in patients with rheumatoid arthritis. Semin Arthritis Rheum. 2009;38(5):366-71.

22. van Sijl AM, Peters MJ, Knol DK, de Vet HC, Gonzalez-Gay MA, Smulders YM, Dijkmans BA, Nurmohamed MT. Carotid intima media thickness in rheumatoid arthritis as compared to control subjects: a meta-analysis. Semin Arthritis Rheum. 2010;40(5):389-97.

23. Rollefstad S, Ikdahl E, Hisdal J, Olsen IC, Holme I, Hammer HB, Smerud KT, Kitas GD, Pedersen TR, Kvien TK, et al. Rosuvastatin-induced carotid plaque regression in patients with inflammatory joint diseases: the Rosuvastatin in Rheumatoid Arthritis, Ankylosing Spondylitis and Other Inflammatory Joint Diseases Study. Arthritis Rhematol. 2015;67(7):1718-28.

24. van den Oever IA, van Sijl AM, Nurmohamed MT. Management of cardiovascular risk in patients with rheumatoid arthritis: evidence and expert opinion. Ther Adv Musculoskelet Dis. 2013:5(4):166-81.

25. Gonzalez-Juanatey C, Llorca J, Testa A, Revuelta J, Garcia-Porrua C, Gonzalez-Gay MA. Increased prevalence of severe subclinical atherosclerotic findings in long-term treated rheumatoid arthritis patients without clinically evident atherosclerotic disease. Medicine (Baltimore). 2003;82(6):407-13.

26. Wartolowska K, Hough MG, Jenkinson M, Andersson J, Wordsworth BP, Tracey I. Structural changes of the brain in rheumatoid arthritis. Arthritis Rheum. 2012;64(2):371-9.

27. Bekkelund SI, Pierre-Jerome C, Husby G, Mellgren SI. Quantitative cerebral MR in rheumatoid arthritis. AJNR Am J Neuroradiol. 1995:16(4):767-72.

\section{Submit your next manuscript to BioMed Central and we will help you at every step:}

- We accept pre-submission inquiries

- Our selector tool helps you to find the most relevant journal

- We provide round the clock customer support

- Convenient online submission

- Thorough peer review

- Inclusion in PubMed and all major indexing services

- Maximum visibility for your research

Submit your manuscript at www.biomedcentral.com/submit
Biomed Central 